\title{
Wolff-Parkinson-White Syndrome Presenting After a Motorcycle Accident
}

\author{
Halil Kaya ${ }^{\mathrm{a}}$, Ozgur Sogut ${ }^{\mathrm{a}, \mathrm{b}}$, Mehmet Tahir Gokdemir ${ }^{\mathrm{a}}$, Mehmet Akif Dokuzoglu ${ }^{\mathrm{a}}$
}

\begin{abstract}
Wolff-Parkinson-White (WPW) syndrome is uncommon in emergency services, but clinicians come across patients with pre-excitation syndrome. WPW is characterized by the presence of an accessory pathway that predisposes patients to tachyarrhythmias and sudden death. Patients with WPW syndrome are potentially at an increased risk of dangerous ventricular arrhythmias. Most of the patients are young and do not have underlying structural heart disease. Management of asymptomatic patients with WPW syndrome has always remained controversial. Here, we present a rare case of a 29-year-old man with WPW syndrome after a motorcycle accident.
\end{abstract}

Keywords: Motorcycle accident; Pre-excitation; WPW syndrome

\section{Introduction}

In 1930, Wolff, Parkinson and White described a series of young patients who had a bundle branch block pattern on electrocardiography (ECG), a short PR interval, and paroxysms of tachycardia [1]. Pre-excitation was defined by Durrer et al. in 1970 as follows: "Pre-excitation exists, if in relation to atrial events, the whole or some part of the ventricular muscle is activated earlier by the impulse originating from the atrium than would be expected if the impulse reached the ventricles by way of the normal specific conduction system only” [2]. Wolff-Parkinson-White (WPW) syndrome

Manuscript accepted for publication September 19, 2011

${ }^{a}$ Harran University, Faculty of Medicine, Department of Emergency Medicine, Sanliurfa, Turkey

bCorresponding author: Ozgur Sogut, Email: drosogut@harran.edu.tr

doi:10.4021/jmc331w is defined as a congenital abnormality involving the presence of abnormal conductive tissue between the atria and the ventricles in association with supraventricular tachycardia (SVT). It involves pre-excitation, which occurs because of the conduction of an atrial impulse, not by means of the normal conduction system, but via an extra atrioventricular (AV) muscular connection [termed an accessory pathway (AP)] that bypasses the AV node [3].

Electrocardiographic findings of WPW syndrome consist of pre-excitation manifested by a shortened PR interval (< $120 \mathrm{~ms}$ ), a widened QRS complex (> $100 \mathrm{~ms}$ ), a delta wave (abnormal initial QRS vector), and SVT. In western countries, the prevalence of WPW syndrome is 1.5-3.1 per 1000 persons [4]. WPW is the second most common cause of paroxysmal SVT in the western world and is the most common cause in China [5]. WPW syndrome may present with palpitations, presyncope, syncope, or sudden cardiac death (SCD). We describe an atypical presentation of WPW syndrome in a young adult following a motorcycle accident.

\section{Case Report}

A 29-year-old man presented to the emergency department complaining of $1 \mathrm{~h}$ of palpitation and fluttering shortage of breath after a motorcycle accident. The patient had no history of intermittent palpitations and a diagnosis of cardiac disorder.

The patient presented with the following vital signs: heart rate $235 \mathrm{bpm}$, arterial blood pressure 120/70 mmHg, and body temperature $36.5^{\circ} \mathrm{C}$. The patient was alert and oriented, and the skin was warm and dry. Pupils were isochoric; direct and indirect pupil light reflexes were positive in both eyes. On cardiac examination, S1 and S2 were normal and there was no heart murmur. Physical examination of the patient was normal, except for right ulna and radius fracture due to the traffic accident. The patient was not taking any medications, nor had he had any follow-up since diagnosis of WPW. He denied cigarette or marijuana smoking and taking cocaine. There was no jugular venous distension. He had tachycardia and shortness of breath, but there was no wheezing or rales. His right wrist was tender, which caused 




Figure 1. Patient's initial ECG. Ventricular rate was $235 \mathrm{bpm}$ and the rhythm was SVT.

discomfort. We applied a short arm splint for his fractures. Physical examination of other systems was unremarkable.

After obtaining successive strips of ECG, the patient was diagnosed with SVT (Fig. 1). His ventricular rate was $235 \mathrm{bpm}$. Within $10 \mathrm{~min}$ of administration of $150 \mathrm{mg}$ amiodarone, the ventricular rate decreased and converted to WPW syndrome rhythm. ECG was consistent with pre-excitation, which was manifested by a shortened PR interval $(<120$ $\mathrm{ms}$ ), a widened QRS (> $100 \mathrm{~ms}$ ), and a delta wave (abnormal initial QRS vector) (Fig. 2). He was then managed by oral propafenone, low-dose aspirin, a histamine $\mathrm{H} 2$ blocker, and a non-steroidal anti-inflammatory drug.

\section{Discussion}

WPW syndrome is found in persons of all ages. Most patients with WPW syndrome present during infancy. However, a second peak of presentation is noted in school-aged children and adolescents. In patients with abnormal ECG findings indicative of WPW syndrome, the frequency of SVT paroxysms increases from $10 \%$ in people aged $20-39$ years to $36 \%$ in people older than 60 years [6]. Overall, about $50 \%$ of patients with WPW develop tachyarrhythmias. In our case, the 12-lead ECG on admission showed SVT with a heart rate of 250 per minute. The incidence of SCD in patients with WPW syndrome has been estimated to range from $0.15 \%$ to $0.39 \%$ [7]. Risk factors for sudden death include, a short pre-excited R-R interval ( $<250 \mathrm{~ms}$ ) during sponta- neous or induced atrial fibrillation (AF), history of symptomatic tachycardia, multiple accessory pathways, Ebstein's anomaly, and familial WPW syndrome [7, 8]. Our patient had none of these risk factors; however, symptomatic tachycardia was observed after the traffic accident. Sudden death may be the first, although infrequent, clinical manifestation of WPW syndrome; therefore, conservative therapy policy is under discussion, and it has been proposed that all patients should receive intervention [8]. Response to long-term antiarrhythmic therapy for the prevention of further episodes of tachycardia in patients with WPW syndrome remains variable and unpredictable. Current diagnostic modalities are accurate in identifying patients with WPW syndrome, but lack the sensitivity to predict SCD [9]. Asymptomatic patients require periodic observation. The onset of cardiac arrhythmias, and possibly the sudden death risk, may be eliminated by prophylactic catheter ablation [7, 9]. We suggest that our patients should also receive prophylactic catheter ablation.

In most patients, SVT is well tolerated and is not lifethreatening. However, the potential for syncope, hemodynamically compromising rhythms, or sudden death may prevent patients with WPW syndrome from participating in competitive sports or dangerous occupations until the substrate is definitively addressed and cured by catheter ablation $[6,7]$.

In a study by Timmermans et al. [8], ventricular fibrillation was the first manifestation of WPW syndrome in 53\% of patients. Rinne et al. [9] presented evidence favoring invasive electrophysiological testing in all patients with WPW 


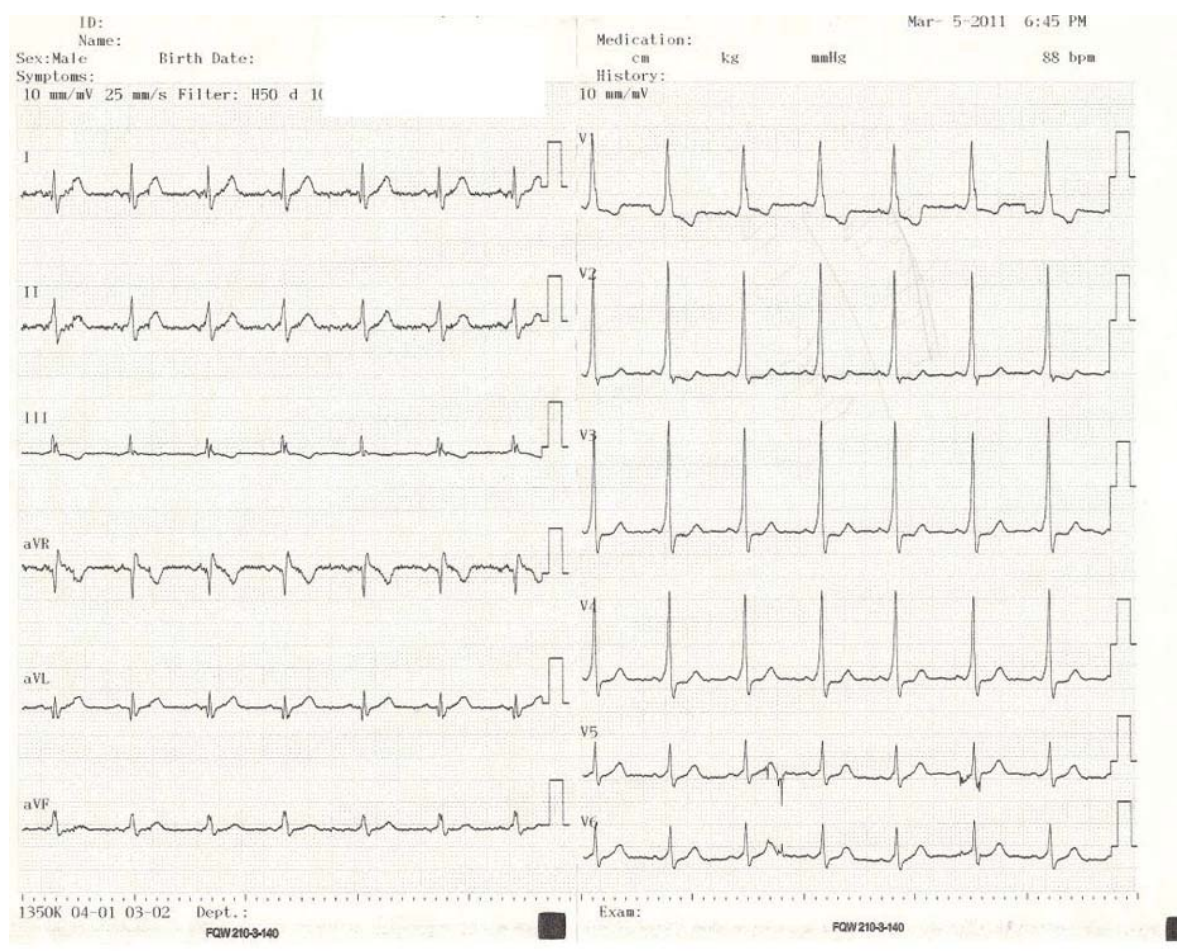

Figure 1. Within $10 \mathrm{~min}$ of administration of $150 \mathrm{mg}$ amiodarone, the ventricular rate decreased and converted to WPW syndrome rhythm. ECG consisted of pre-excitation manifested by a shortened PR interval (<120 ms), a widened QRS (> $100 \mathrm{~ms}$ ), and a delta wave (abnormal initial QRS vector).

syndrome and palpitations.

WPW syndrome is commonly diagnosed on the basis of the surface ECG in an asymptomatic individual [6, 9]. In this case, it is manifested as a delta wave, which is a slurred upstroke in the QRS complex that is associated with a short PR interval. The short PR interval and slurring of the QRS complex is actually the impulse making it through to the ventricles prematurely (across the AP), without the usual delay experienced in the $\mathrm{AV}$ node.

If the patient experiences episodes of AF, the ECG will show a rapid polymorphic wide-complex tachycardia (without torsades de pointes). This combination of AF and WPW is considered dangerous, and most antiarrhythmic drugs are contraindicated. When an individual is in normal sinus rhythm, the ECG characteristics of WPW syndrome are a short PR interval, widened QRS complex (> $120 \mathrm{~ms}$ in length) with slurred upstroke of the QRS complex, and secondary repolarization changes reflected in ST segment- $\mathrm{T}$ wave changes [9, 10]. Similarly, our case had a short PR interval, widened QRS complex (> $120 \mathrm{~ms}$ in length) with slurred upstroke of the QRS complex, and secondary repolarization changes reflected in ST segment-T wave changes.

In the case of type A pre-excitation (left AV connections), a positive R wave is seen in V1 ("positive delta") on the precordial leads of the electrocardiogram, while in type B pre-excitation (right AV connections), a predominantly negative delta wave is seen in lead V1 ("negative delta”)
[6]. Due to the positive R wave seen in V1 ("positive delta") on the precordial leads of the electrocardiogram, the present case was considered to be a type A WPW.

Treatment of tachycardia associated with WPW is similar to treat paroxysmal SVT, with a focus on breaking the cyclical transmission of impulses. This is best accomplished by temporarily prolonging the refractory period of the AV node with drugs such as adenosine [10]. The most favorable plan is to treat symptomatic WPW syndrome patients with ablation to cure the tachycardia and eliminate the potential dangerous effects of drugs [7, 10]. Electrophysiologic study (EPS) was planned for the present case.

Acutely, people with WPW who are experiencing tachyarrhythmias may require synchronized electrical cardioversion if their condition is critical (e.g., they are hypotensive or lethargic with altered mental status); if they are stable, medical treatment may be used [11]. Patients with $\mathrm{AF}$ and rapid ventricular response are often treated with amiodarone or procainamide to stabilize their heart rate. Procainamide, amiodarone, and cardioversion are now accepted treatments for the conversion of tachycardia found with WPW. Adenosine and other AV node blockers including diltiazem, verapamil, other calcium channel blockers and beta blockers should be avoided in AF and atrial flutter with WPW $[9,11]$. The definitive treatment of WPW syndrome is destruction of the abnormal electrical pathway by radiofrequency catheter ablation [7, 11]. 


\section{Conclusion}

Early diagnosis, correct treatment, and patient education are of principal importance in patients with WPW syndrome. Patients should be referred for catheter ablation, and conduction abnormalities (e.g., AV block or slowing of conduction) can be handled clinically with the placement of a permanent pacemaker. Periodic follow-up care of patients is necessary, along with consideration for EPS and prophylactic catheter ablation. This is especially true in asymptomatic young patients who have been told of their abnormal ECG results.

\section{References}

1. Wolff L, Parkinson J, White PD. Bundle-branch block with short P-R interval in healthy young people prone to paroxysmal tachycardia. 1930. Ann Noninvasive Electrocardiol. 2006;11(4):340-353.

2. Durrer D, Schuilenburg RM, Wellens HJ. Pre-excitation revisited. Am J Cardiol. 1970;25(6):690-697.

3. Calkins H, Sousa J, el-Atassi R, Rosenheck S, de Buitleir M, Kou WH, Kadish AH, et al. Diagnosis and cure of the Wolff-Parkinson-White syndrome or paroxysmal supraventricular tachycardias during a single electrophysiologic test. N Engl J Med. 1991;324(23):1612-1618.

4. Guize L, Soria R, Chaouat JC, Chretien JM, Houe D, Le Heuzey JY. Prevalence and course of Wolf-ParkinsonWhite syndrome in a population of 138,048 subjects. Ann Med Interne (Paris). 1985;136(6):474-478.
5. Wan Q, Wu N, Fan W, Tang YY, Jin L, Fang Q. Clinical manifestations and prevalence of different types of supraventricular tachycardia among Chinese. Chin Med J (Engl). 1992;105(4):284-288.

6. Brembilla-Perrot B, Yangni N'da O, Huttin O, Chometon F, Groben L, Christophe C, Benzaghou N, et al. Wolff-Parkinson-White syndrome in the elderly: clinical and electrophysiological findings. Arch Cardiovasc Dis. 2008;101(1):18-22.

7. Pappone C, Santinelli V, Manguso F, Augello G, Santinelli O, Vicedomini G, Gulletta S, et al. A randomized study of prophylactic catheter ablation in asymptomatic patients with the Wolff-Parkinson-White syndrome. N Engl J Med. 2003;349(19):1803-1811.

8. Timmermans C, Smeets JL, Rodriguez LM, Vrouchos G, van den Dool A, Wellens HJ. Aborted sudden death in the Wolff-Parkinson-White syndrome. Am J Cardiol. 1995;76(7):492-494.

9. Rinne C, Klein GJ, Sharma AD, Yee R, Milstein S, Rattes MF. Relation between clinical presentation and induced arrhythmias in the Wolff-Parkinson-White syndrome. Am J Cardiol. 1987;60(7):576-579.

10. Sharma AD, Klein GJ, Yee R. Intravenous adenosine triphosphate during wide QRS complex tachycardia: safety, therapeutic efficacy, and diagnostic utility. Am J Med. 1990;88(4):337-343.

11. Fengler BT, Brady WJ, Plautz CU. Atrial fibrillation in the Wolff-Parkinson-White syndrome: ECG recognition and treatment in the ED. Am J Emerg Med. 2007;25(5):576-583. 